\title{
Packing and Characterizing High- power Laser Diode
}

\author{
Nguyen Tuan Anh ${ }^{1,}$, Didier Decoster ${ }^{2}$, Au Thi Huong ${ }^{2}$ \\ ${ }^{l}$ NACENTECH, 25 Le Thanh Tong, Hoan Kiem, Hanoi, Vietnam \\ ${ }^{2}$ PolyTech lille, University Lille 1, France \\ Received 16 August 2016 \\ Revised 15 September 2016; Accepted 09 September 2016
}

\begin{abstract}
In the manufacturing processes of high-power laser diode modules, the module packaging technologies play an important role; decide the efficiency of the electro-optical conversation, the beam quality and the lifetime. This paper presents the packaging of high-power laser diode modules from single chip, evaluating the electro-optical characteristics and the beam quality of the modules. The obtained results show that the packaged module has an output optical power of $5 \mathrm{~W}$ at current $5.9 \mathrm{~A}$; the Operation Voltage is $1.7 \mathrm{~V}$; the Threshold Current is $1,0 \mathrm{~A}$; the Divergence Angle Mean is 36.8 degree and the Asymmetry is 1.04. These parameters are almost the same that of some on-stock modules. Thus, the application of the packaged module in social economics is possible.
\end{abstract}

Keywords: Laser diode, fiber - coupled, die bond technology; wire bond technology; electrooptical conversation efficiency, PVI characteristics, beam quality, Divergence Angle, Asymmetry.

\section{Introduction}

The revolution of semiconductor laser technology in the last decades has made the adoption of high-power laser diodes more affordable [1]. The advantages of high-power laser diodes in terms of compactness, energy efficiency, and lifetime and running costs have been increasingly recognized. Nowadays, high-power laser diodes are sufficient for many different applications such as pumping of solid state lasers, material processing, medical applications, the successful applications of high-power diode lasers depend on their high reliability in combination with a long lifetime.

However, the packaging of a high-power laser diode is time and effort consuming. The consideration places not only on the thermal challenges, the mechanical integrity, the electrical coupling, the excessively-induced bonding stress, but also on the optical stringent alignment in order to achieve high optical coupling between the very small facet areas (few microns square) with the pigtail fibre.

At the moment, the actual development of high-power laser diodes is governed by two trends: the trend of packaging high-power laser bar [2,3] and the trend of packaging high-power laser diodes from single chip $[4,5]$.

\footnotetext{
* Corresponding author. Tel.: 84- 912442705

Email: cfoctuananh@yahoo.com
} 
The advantage of the first approach is that the optical power of laser bars is high. Nevertheless, it requires an efficient heat sink to keep the temperature of the active zone of the laser diode bar low. Normally, the water cooling methods are used.

At the second approach, there are some different methods but the typical one is shown in Fig. 1, where laser diode chip is bonded on a substrate, which is then placed onto a heat sink. A highly conductive material, such as indium is placed between the substrate and the heat sink. Sometimes, a thermistor (either placed on the heat sink or bonded to the substrate) is used in combination with TEC to regulate the temperature of the laser.

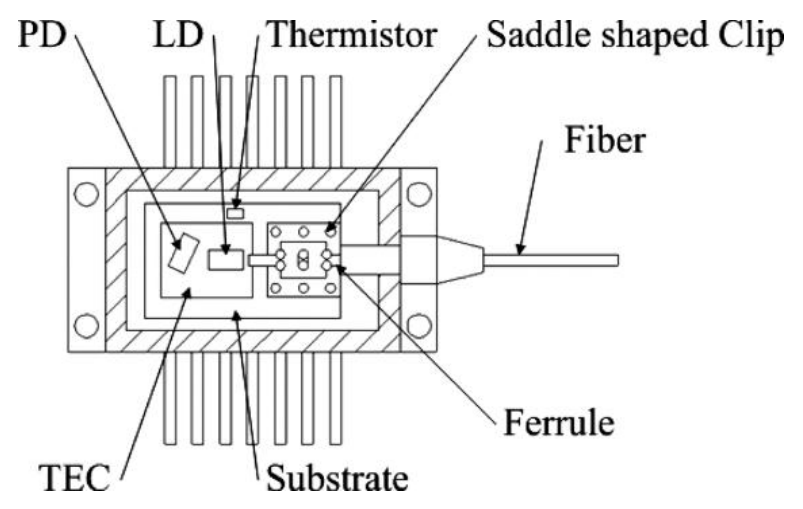

Fig. 1. A schematic diagram of a single chip laser diode module

In the manufacturing companies, for packaging high-power laser diodes, they normally use a combination of different technological equipment such as a Clean Room, Die/Wire Bonders, Optical Table Systems, Alignment Stages, Probe Systems, Current Controller, Temperature Controller, Optical Power Meters, Monitoring Systems, ...

In Vietnam, we meet many difficulties to package high-power laser diodes. There are many reasons but mainly due to the lack of packaging equipment. Therefore, most of recent researches related to laser diodes are either low-power laser diodes or the application of imported laser diodes.

Fortunately, in the past few years, the National Centre for Technological Progress, the Ministry of Science and Technology has been equipped with a number of specialized packaging equipment that allows to package high-power laser diode modules.

In this paper, the authors will present methods of packaging high-power laser diodes from single chip and evaluate the electro-optical characteristics of the packaged laser diode modules.

\section{Packaging high-power laser diodes}

\subsection{Die bonding}

In our experiment, we follow the method of packaging high-power laser diodes from single chip. The "p-down" laser chip is mounted on the copper heat sink by the way that p-side is well contacted with the heat sink.

To avoid the induction of thermo-mechanical stress to the chip, a thin indium layer is placed between p-contact and the heat sink (Fig. 2) before mounting the chip on the heat sink. Both surfaces of the chip and the heat sink are well polished in a Clean Room before placing the indium layer. 


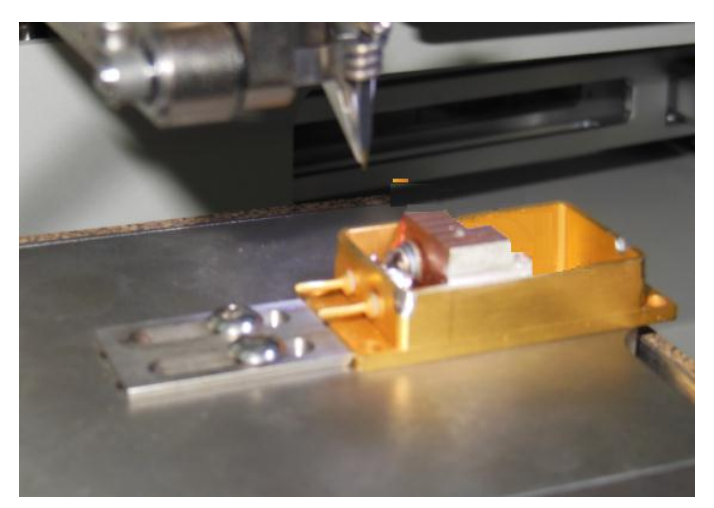

Fig. 2. The laser diode is mounted on the copper heat sink.

In die bonding technique, there are three essential parameters determining the quality of die bonding. They are the chip picked-up force, the on-chip pressure and the bonding temperature. The chip pick-up force is the force acting on chip when it is picked up. The on-chip pressure is the force presses on chip during bonding. The bonding temperature is the temperature applied to the die bonding.

The selection of appropriate parameters for chip picked-up force, the on-chip pressure and the bonding temperature is extremely important. Experiment with these parameters is time consuming, and is an important step toward developing a die bonding process.

In our experiment, the 7372E Wesbond die bonding equipment is used during the die bounding process. After many trials, we have established the optimal parameters for a single-chip die bonding as in table 1 .

Table 1. Parameters of die bonding

\begin{tabular}{lllll}
\hline Parameters & Unit & Min Value & Mean Value & Max Value \\
\hline chip picked-up force & $\mathrm{g}$ & 100 & 150 & 200 \\
on-chip pressure & $\mathrm{g}$ & 150 & 200 & 250 \\
bonding temperature & ${ }^{0} \mathrm{C}$ & 150 & 200 & 250 \\
\hline
\end{tabular}

\subsection{Wire bonding}

To provide the electrical paths for power and signal distribution, the chip-to-substrate interconnection is tacken into place. The most common interconnecting method is wire bonding (Fig. 3).

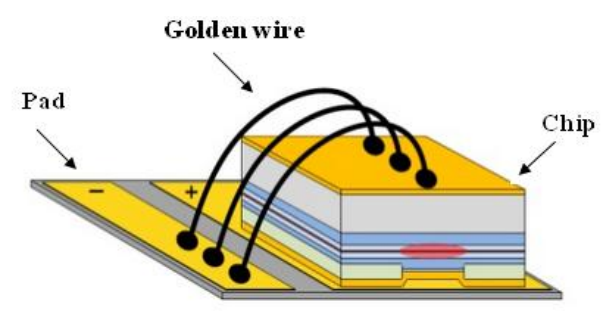

Fig. 3. Interconnection inside the laser diode modules. 
Wire bonding is an electrical interconnection technique using thin gold wire and a combination of compression force, ultrasonic frequency, ultrasonic power and gold wire diameter. Wire bonding is a solid phase welding process, where the two metallic materials (wire and the pad surface) are brought into common contact. Once the surfaces are in common contact, electron sharing or inter diffusion of atoms takes place, resulting in the formation of a bond. The compression force initially deforms the wire and couples the ultrasonic energy into the wire and the pad. Optimizing a wire bond process begins with a clear understanding of the bonding equipment, machine set-up, the response variables involved, and their relationship to one another.

Similar to the die bonding technique, setting the optimal condition for wire bonding places a key role to ensure the quality of the wire bonding. In our experiment, the wire bounding is done by $7476 \mathrm{D}$ Westbound wire bonding equipment (Fig. 4). The $50 \mu \mathrm{m}$ gold wire is used for the wire bounding process.

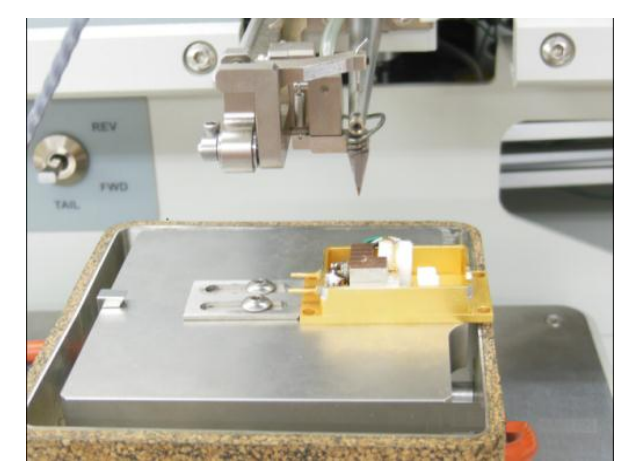

Fig. 4. The laser diode is wire-bonded by $7476 \mathrm{D}$ Westbound.

After many trials, we have established the optimal parameter table for wire bonding of single chip laser diode as shown in table 2.

Table 2. Parameters of wire bonding

\begin{tabular}{lllll}
\hline Parameters & Unit & Min Value & Mean Value & Max Value \\
\hline compression force & $\mathrm{g}$ & 50 & 75 & 100 \\
ultrasonic frequency & $\mathrm{kHz}$ & 63 & \\
ultrasonic power & $\mathrm{W}$ & 4 & \\
wire diameter & $\mu \mathrm{m}$ & 50 & \\
\hline
\end{tabular}

\subsection{Optical coupling}

In many applications, the laser beam must be focused and transmitted into optical fiber. To do this, normally in the production line, one must use multiple dedicated devices such as optical tables, table alignment, probe systems, current controller, temperature controller, optical power meter, vision systems,...

This is one of the most time and effort consuming stages in laser diode packaging process. The relative position of the laser diode and the heat sink plays an important role in optical coupling. It is required to precisely align the laser facet (with few microns square area) and the pigtail fiber in order to couple the light into the fiber.

The schematic of the optical coupling is shown in Fig. 5. 


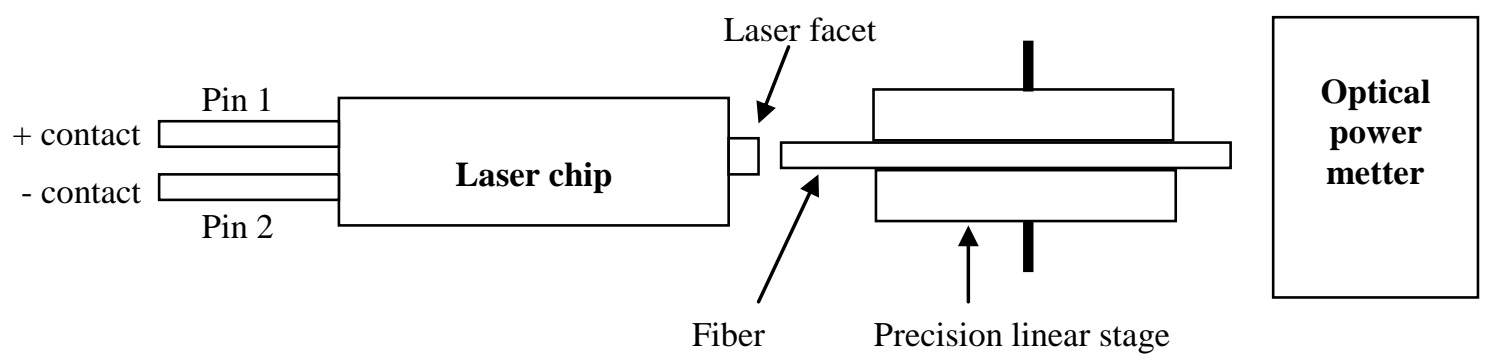

Fig. 5. Schematic of the optical coupling.

A $105 \mu \mathrm{m}$ multimode fiber is placed on a precision linear stage. One end of the fiber leads to an optical power meter, the other end is precisely aligned with the laser facet. Current of $1 \mathrm{~A}$ from the Newport current laser driver 560B is applied to the laser. The well aligned position is obtained when optical power gets its maximum value. Newport optical power meter 1916C in combination with Newport optical detector $818 \mathrm{P}$ is used for this measurement. Fig. 6 shows the image during the process of optical coupling.

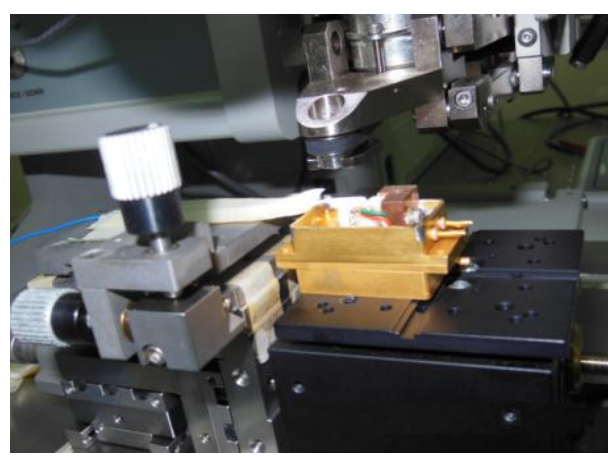

Fig. 6. Image during the process of optical coupling.

\subsection{Module packaging}

This is the last step in the process of packaging laser module. After finishing the die bonding, wire bonding and fiber coupling, all these parts are sealed in clean environment. The module substrate is coated with a special epoxy before being covered with the lid. Fig. 7 shows some images during the process.

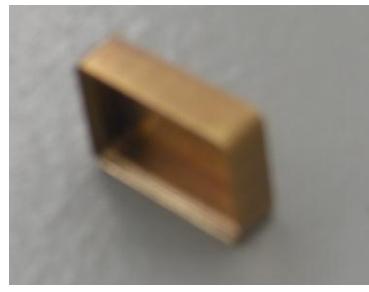

a)

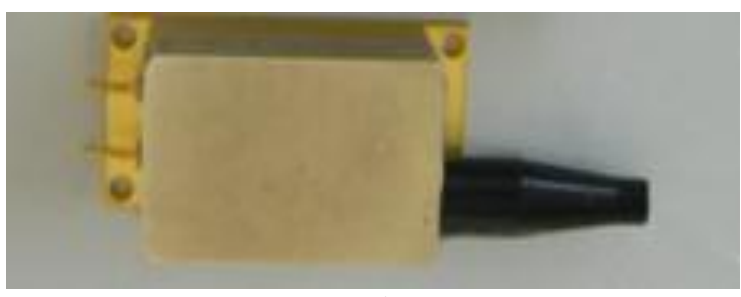

b)

Fig. 7. Some images during the process of module packaging: (a) the lid of laser diode module; (b) image of the completed laser diode module. 


\section{Characteristics of high-power laser diodes}

To evaluate the quality of the packaged laser diode modules, some characteristics such as electrooptical conversation: V-I, P-I, and the beam quality are interested.

\subsection{V-I characteristic and Operation Voltage}

V-I Characteristic represent the relationship between the voltage applied to the laser module and the current. The laser module is driven by a current source model 560B Newport. The measurements were carried out in laboratory conditions $\left(\right.$ at $25^{\circ} \mathrm{C}$ ). The current is changed in steps of $50 \mathrm{~mA}$. Voltage applied to the module is measured by multimeter Sanwa PC510A.

At each current value, the measurement is performed three times. Measurement results are the average values of these three measurements. The measurement results are listed in table 3 .

Table 3. The relationship between voltage and current of the packaged laser module

\begin{tabular}{llll}
\hline $\begin{array}{l}\text { Current } \\
(\mathrm{mA})\end{array}$ & $\begin{array}{l}\text { Mean Voltage } \\
(\mathrm{mV})\end{array}$ & $\begin{array}{l}\text { Current } \\
(\mathrm{mA})\end{array}$ & $\begin{array}{l}\text { Mean Voltage } \\
(\mathrm{mV})\end{array}$ \\
\hline 0 & 0 & 750 & 1350 \\
50 & 90 & 800 & 1440 \\
100 & 180 & 850 & 1530 \\
150 & 270 & 900 & 1600 \\
200 & 360 & 950 & 1650 \\
250 & 450 & 1000 & 1680 \\
300 & 540 & 1050 & 1690 \\
350 & 630 & 1100 & 1700 \\
400 & 720 & 1150 & 1705 \\
450 & 810 & 1200 & 1710 \\
500 & 900 & 1250 & 1715 \\
550 & 990 & 1300 & 1720 \\
600 & 1080 & 1350 & 1725 \\
650 & 1170 & 1400 & 1730 \\
700 & 1260 & 1450 & 1735 \\
\hline
\end{tabular}

From table 3, it is easy to plot the V-I as depicted in Fig. 7.

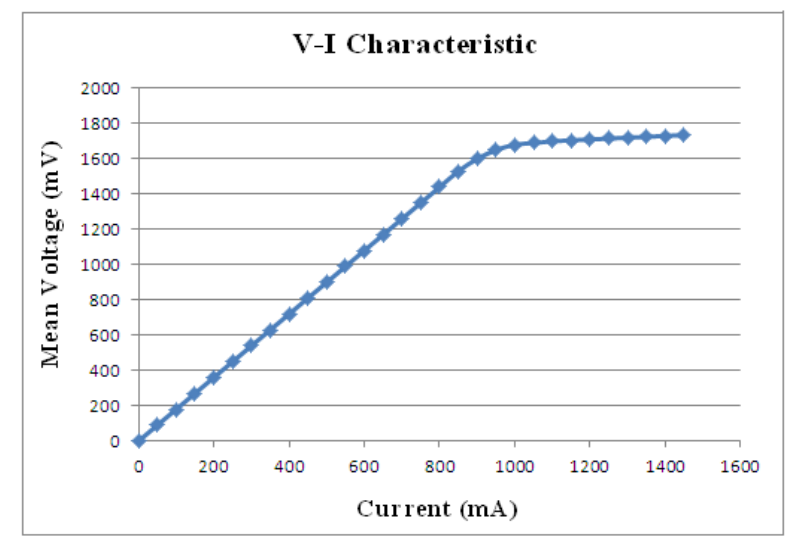

Fig. 7. V-I Characteristic of the packaged laser diode module. 
From table 3 and the V-I characteristic, one can see that the Operating Voltage of the packaged laser diode module is about $1.7 \mathrm{~V}$.

\subsection{P-I Characteristic and the Threshold Current}

P-I characteristic represent the relationship between the optical power and the applied current. The optical output power is plotted versus the driving current. In this measurement, Newport laser driver source 560B, the Newport power meter 1916C and Newport optical power detector 818P are used. Current applied to the laser diode increases from $0 \mathrm{~mA}$ to $5.5 \mathrm{~A}$ by a step of $100 \mathrm{~mA}$. At each current value, power reading is recorded (Fig. 8).

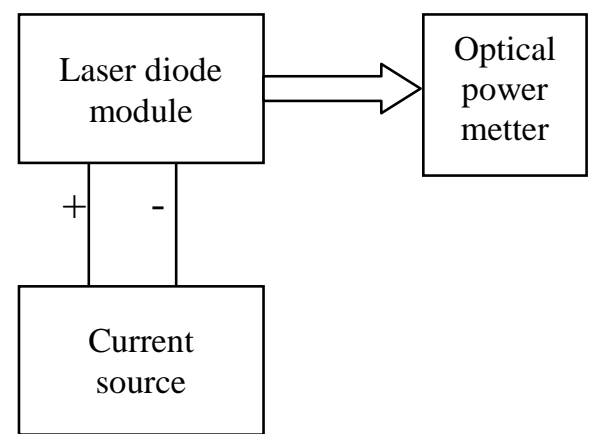

a)

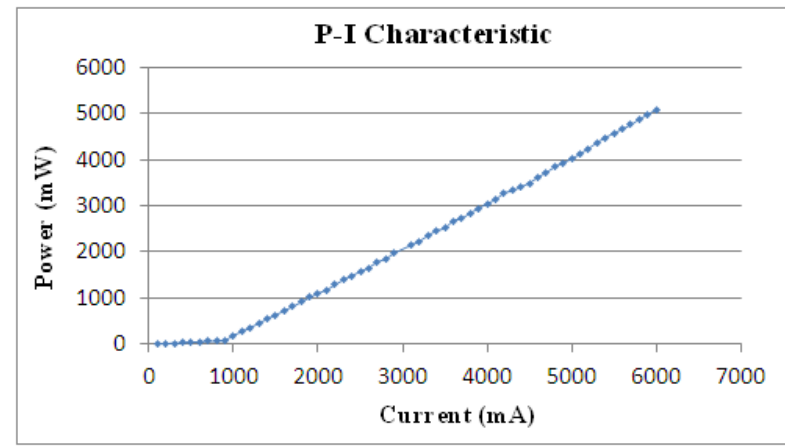

b)

Fig. 8. The optical power - Current measurement: a - Schematic of the measurement; b- Optical powerCurrent characterization of the laser diode

Measurement results are recorded and listed in table 4.

Table 4. The relationship between Optical Power and Current

\begin{tabular}{llll}
\hline Current $(\mathrm{mA})$ & $\begin{array}{l}\text { Optical Power } \\
(\mathrm{mW})\end{array}$ & Current $(\mathrm{mA})$ & $\begin{array}{l}\text { Optical Power } \\
(\mathrm{mW})\end{array}$ \\
\hline 100 & 0 & 3200 & 2235 \\
200 & 10 & 3300 & 2355 \\
300 & 18 & 3400 & 2460 \\
400 & 25 & 3500 & 2515 \\
500 & 40 & 3600 & 2675 \\
600 & 50 & 3700 & 2725 \\
700 & 62 & 3800 & 2845 \\
800 & 65 & 3900 & 2945 \\
900 & 84 & 4000 & 3050 \\
1000 & 190 & 4100 & 3135 \\
1100 & 270 & 4200 & 3275 \\
1200 & 360 & 4300 & 3325 \\
1300 & 450 & 4400 & 3410 \\
1400 & 550 & 4500 & 3495 \\
1500 & 630 & 4600 & 3620 \\
1600 & 730 & 4700 & 3723 \\
1700 & 810 & 4800 & 3845 \\
1800 & 910 & 4900 & 3935 \\
1900 & 1010 & 5000 & 4015 \\
\hline
\end{tabular}




\begin{tabular}{llll}
\hline 2000 & 1100 & 5100 & 4130 \\
2100 & 1175 & 5200 & 4242 \\
2200 & 1285 & 5300 & 4375 \\
2300 & 1395 & 5400 & 4482 \\
2400 & 1480 & 5500 & 4578 \\
2500 & 1575 & 5600 & 4663 \\
2600 & 1655 & 5700 & 4758 \\
2700 & 1780 & 5800 & 4875 \\
2800 & 1845 & 5900 & 4983 \\
2900 & 1970 & 6000 & 5085 \\
3100 & 2145 & & \\
\hline
\end{tabular}

One can see from Fig. $8 \mathrm{~b}$ that the threshold current can be calculated from the Optical powerCurrent curve. As indicated in Fig.8b, threshold current of the laser diode is around $1 \mathrm{~A}$.

\subsection{Energy distribution of the laser beam}

To evaluate the quality of the laser beam, the energy distribution of the laser beam is measured by Thorlabs Beam Analyzing BP 109 - IR equipment. As the laser chip has a center wavelength of 940 $\mathrm{nm}$, this equipment is set at the wavelength of $940 \mathrm{~nm}$. The measured results are shown in Fig. 9.

The parameters and measured values are displayed on the screen and stored as data files. Energy spectrum of the beam is described in Fig. 9, where $\mathrm{Y}$ axis is the relative values calculating in \%, compared with the maximum value. $\mathrm{X}$ axis is the distance to both sides of the maximum power value.

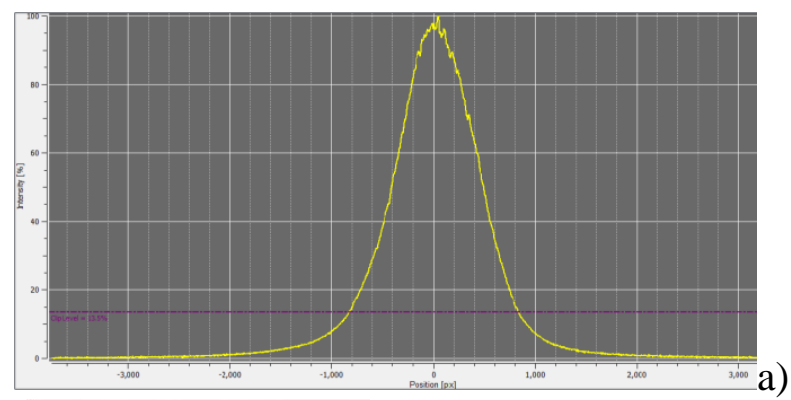

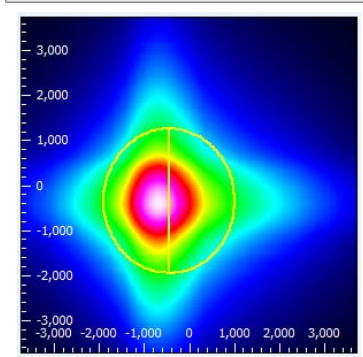

b)

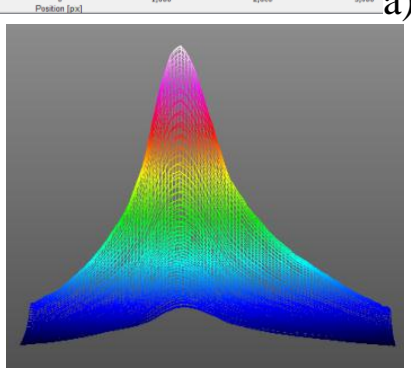

c)

Fig. 9. Distribution of laser beam energy: a) relative value of the beam energy in $\%$ compared to the maximum value; b) energy contours; c) 3D Image.

From the Fig. 9, we can see that the energy distribution has the form of Gaussian beam. This means that we can approximately apply calculations on the laser beam of the packaged module as in the case of Gaussian beam. Moreover, the spot size of the laser beam is roughly circular shape. This proves that the beam quality is relatively good. 


\subsection{Divergence angle and Asymmetry measurement}

Laser diode emission is confined to a narrow p-n junction region, thus, diffraction of the laser beam results in a large divergence. Half-angle beam divergence $\theta$ is given by [6]:

$$
\theta \sim \sin ^{-1}(\lambda / a)
$$

where $\mathrm{a}$ is the width of active region and $\lambda$ is the laser wavelength.

In practice, the beam divergence angle is generally determined by the beam cross section [7]. Generally, the cross section with elliptical beam, the beam divergence angles are determined by both the axis of the ellipse. However, in exceptional cases, when the beam cross section is circular, the divergence angle can be determined by the formula:

$$
\Theta=2 \arctan \left(\frac{D_{f}-D_{i}}{2 l}\right)
$$

where $D_{f}$ and $D_{i}$ are two positions in the beam propagation direction and $l$ is the distance between the two these positions.

In the special case with the Gaussian shaped beam, half divergence angle, $\theta$, is determined by the formula [7]:

$$
\theta=\frac{\lambda}{\pi \omega}
$$

where $\lambda$ is the wavelength, $\omega$ is the beam waist (smallest cross section of the beam).

As mentioned above, the beam of the packaged module is considered to have Gaussian form. Therefore, we can apply the formula for the Gaussian beam to calculate the beam divergence angle. To determine the divergence angle as well as the asymmetric, we have set up the experiment on Thorlabs Analyzing BP109-IR Beam. Measurement results are described in table 5.

Table 5. Divergence angle and Asymmetry measurement

\section{Divergence Test Protocol}

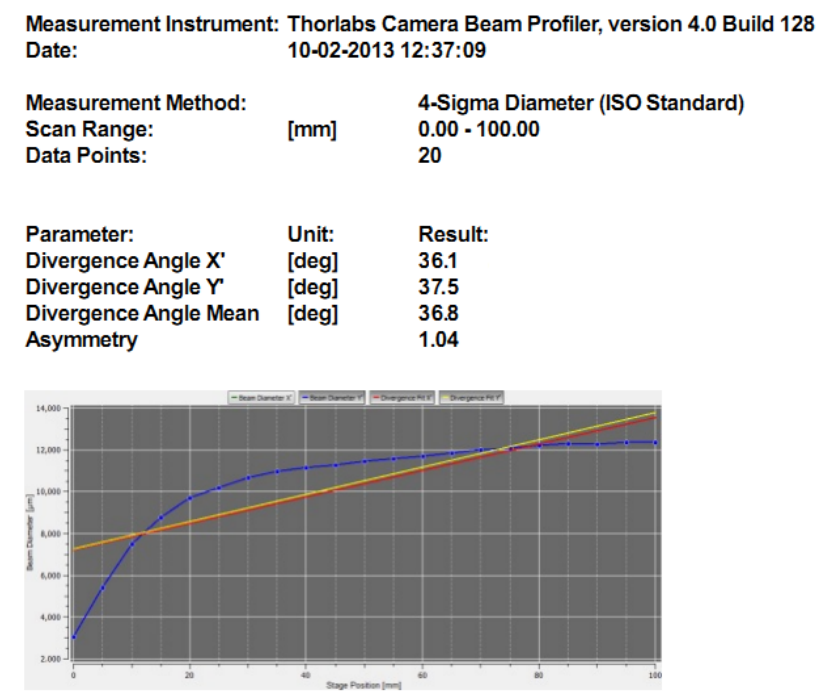


From table 5, we can see that the beam divergence on $\mathrm{X}$ and $\mathrm{Y}$ axes are 36.10 and 37.50, respectively. The average divergence angle is 36,80 and the asymmetry is 1.04 .

To evaluate the beam quality of the packaged laser diode module, the comparison with some onstock modules [8] is taken in place. The compilation data is listed in table 6.

Table 6. Beam quality comparison between packaged modules and on-stock modules

\begin{tabular}{lllll}
\hline \multirow{2}{*}{ No. } & \multirow{2}{*}{ Parameters } & \multirow{2}{*}{ Packaged module } & $\begin{array}{l}\text { AM6-940C-20-458 } \\
\text { ALFALIGHT }\end{array}$ & $\begin{array}{l}\text { WSLD-940-005-3 } \\
\text { WAVESPECTRUM }\end{array}$ \\
\hline 1 & Center wavelength, $\lambda_{\mathrm{c}}$ & $940 \mathrm{~nm}$ & $940 \mathrm{~nm}$ & $940 \mathrm{~nm}$ \\
2 & Operation Current, $\mathrm{I}_{\mathrm{op}} @ 5 \mathrm{~W}$ & $\sim 5,9 \mathrm{~A}$ & $5,4 \mathrm{~A}$ & $5,2 \mathrm{~A}$ \\
3 & Operation Voltage, $\mathrm{V}_{\text {op }}$ & $\sim 1,7 \mathrm{~V}$ & $1,6 \mathrm{~V}$ & $2,0 \mathrm{~V}$ \\
4 & Threshold Current, $\mathrm{I}_{\text {th }}$ & $\sim 1,0 \mathrm{~A}$ & $0,7 \mathrm{~A}$ & $1,0 \mathrm{~A}$ \\
5 & Divergence Angle, $\Theta$ & $36,8^{0}$ & - & $36^{0}$ \\
6 & Asymmetry & 1,04 & - & - \\
\hline
\end{tabular}

From table 6, one can see that the beam quality of the packaged laser module is similar to that of some on-stock laser modules. It means that the high power laser diode packaging and its application in Vietnam is possible.

\section{Conclusions}

Although the performance of high-power laser diodes in terms of output power, laser beam quality and lifetime has increased recently. However, the mounting of these lasers still remains a critical point in the fabrication process of these devices.

With a great attempt, a laser diode module with the center wavelength of $940 \mathrm{~nm}$, the Divergence Angle Mean of 36.8 degree, the Asymmetry of 1.04 and the $\mathrm{CW}$ optical output power of $5 \mathrm{~W}$ is fabricated

Nevertheless, for applications, further measurements such as the lifetime, the stability of the laser module and the on-field trial should be taken into place.

\section{References}

[1] Colin E Webb, Julian D C Jones (2004), handbook of Laser Technology and Applications, Volume II - Laser design and Laser system, IOP Publishing, Bristol and Philadelphia.

[2] Xingsheng Liu, Wei Zhao, Lingling Xiong, Hui Liu (2015), Packaging of High Power Semiconductor Lasers, Springer New York.

[3] Duesterberg, R., et al. (2011), “100W high-brighness multi-emitter laser pump”, Proc. SPIE, 7918.

[4] J. Braunstein, M. Mikulla, R. Kiefer, M. Walther, J. Jandeleit, W. Brandenburg, P. Loosen, R. Poprawe, G. Weimann, (2000), "267 W cw AlGaAs/GaInAs Diode Laser Bars", SPIE Proc. Photonics West, Vol. 3945, San Jose, USA.

[5] K. Wade, L.J. Mawst, D. Botez, (1997), "High continuous wave power, $0.8 \mu$ m-band, Al-free active region diode lasers“, Appl. Phys. Lett., 70 (2), pp. 149-151.

[6] Das P., (1991), “Lasers and optical engineering”, New York: Springer-Verlag.

[7] https://en.wikipedia.org/wiki/Beam_divergence

[8] http://www.laserdiodesource.com/laser-diode-product-page 